\title{
Neutron Production in Extensive Air Showers
}

\section{Ralph Engel, ${ }^{a, b, *}$ Alfredo Ferrari, ${ }^{a, c}$ Markus Roth, ${ }^{a}$ Martin Schimassek, ${ }^{b}$ David Schmidt ${ }^{b}$ and Darko Veberič ${ }^{a}$}

${ }^{a}$ Institute for Astroparticle Physics (IAP),

Karlsruhe Institute of Technology (KIT)

P.O. Box 3640, Karlsruhe, Germany

${ }^{b}$ Institute for Experimental Particle Physics (ETP),

Karlsruhe Institute of Technology (KIT)

P.O. Box 3640, Karlsruhe, Germany

${ }^{c}$ Istituto Nazionale di Fisica Nucleare, Sezione di Milano,

Via Celoria 16, 20133 Milano, Italy

E-mail: ralph.engel@kit.edu, alfredo.ferrari@kit.edu

We use the Monte Carlo simulation package FLUKA to study the production of neutrons in extensive air showers. In contrast to typical shower simulations, we consider the full range of neutron energies extending down to thermal neutrons. The importance of different neutron production mechanisms and their impact on the predicted neutron distributions in energy, lateral distance, and arrival time are discussed and compared with those of muons. In addition, the dependence of the predictions on the primary particle is studied.

$37^{\text {th }}$ International Cosmic Ray Conference (ICRC 2021)

July 12th - 23rd, 2021

Online - Berlin, Germany

\footnotetext{
*Presenter
} 


\section{Introduction}

Neutrons are the only neutral and stable ${ }^{1}$ particles produced in hadronic interactions. Because they don't loose energy by ionization, their propagation and energy loss are solely determined by hadronic interactions and quasi-elastic scattering. Therefore, the neutron cloud of an air shower exhibits features that are different from those of the electromagnetic and muonic shower components.

Already in the early years of air shower measurements, investigations of the neutron component of showers were carried out, see, for example, [1]. Already then it was understood that low-energy neutrons arrive with a considerable time delay with respect to the other shower particles and this delay was used to identify them. In a pioneering paper Linsley, discussed the observation of late pulses, caused by subluminal particles, and estimated the energy content of the neutron component of air showers [2]. Today we have a series of measurements of slow neutrons produced in air showers that were taken with dedicated detectors, see [3,4] and [5] for a discussion. The interpretation of neutron data is, however, hampered by the difficulties to calculate reliable predictions. Typical air shower programs are not built to treat low-energy neutrons.

Motivated by the operation or planned installation of large scintillator arrays, we re-visit the production of neutrons in air showers in this work. In contrast to previous works we use FLUKA [6, 7] for the detailed simulation of air showers and fully account for the production and propagation of neutrons of all energies. In this contribution, we present some general results of these simulations that are independent of possible detection methods. In Sec. 2, we argue that FLUKA is a very well suited tool for performing such a study. The simulation method and first results obtained with it are given in Secs. 3 and 4. Considering vertical showers in the knee energy range, we compare our neutron results to those of muons because the phenomenology of muon production is well understood and provides a good reference.

\section{The FLUKA code}

FLUKA [6, 7] (www.fluka.org) is a fully integrated Monte Carlo simulation package for interaction and transport of particles and nuclei in matter. FLUKA has many applications in particle physics, high energy experimental physics and engineering, shielding, detector design, cosmic ray studies, dosimetry, medical physics, radiobiology, and hadron therapy. Over the last 30 years there has been a multitude of verifications of the accuracy of the FLUKA code in predicting particle production spectra, and in particular neutrons, around high energy accelerators, and by cosmic rays.

FLUKA nuclear reaction models are based on the PEANUT [7, 8] hadron-nucleus generator for projectile energies up to $20 \mathrm{TeV}$ in the laboratory system. At higher hadron energies, the latest development [9] of the DPMJET-III code [10] is used: this code has been extensively benchmarked against LHC data and its predictions are therefore reliable until up to tens of $\mathrm{TeV}$ in the nucleonnucleon center-of-mass. For ion projectiles, DPMJET-III is again used for energies above $5 \mathrm{GeV} / \mathrm{n}$, while at lower energies a vastly modified version of rQMD-2.4 [11] manages nucleus-nucleus interactions. Finally, of minimal relevance for this study, FLUKA uses the BME model [12] for ions below $125 \mathrm{MeV} / \mathrm{n}$. FLUKA can also deal with real and virtual photonuclear interactions, a capability which is critical for the results presented in this paper.

\footnotetext{
${ }^{1}$ Stable on time scales of relevance for air shower physics.
} 
To illustrate the capabilities of the FLUKA code package we compare FLUKA predictions with neutron measurements at different altitudes, see Fig. 1. Two sets of experimental data have been used, the measurements taken aboard an ER-2 airplane at high altitude in 1997 and subsequently at ground level in 1999 [13], and those taken on top the Zugspitze mountain (2963 m), in 1995 [14].

Both experiments measured the neutron intensity and spectra using a Bonner multi-sphere spectrometer. As such, the reported spectra were obtained by unfolding the count readings of the spheres, a procedure which is somewhat dependent on the guess spectrum used in the unfolding procedure, and on the accuracy of the computed response functions of the Bonner spheres. As a consequence, the spectral features above $\approx 20 \mathrm{MeV}$ are those coming from the guess spectra, and the integral intensity of the high energy component is dependent on the accuracy of the codes used for the computation of the response functions.
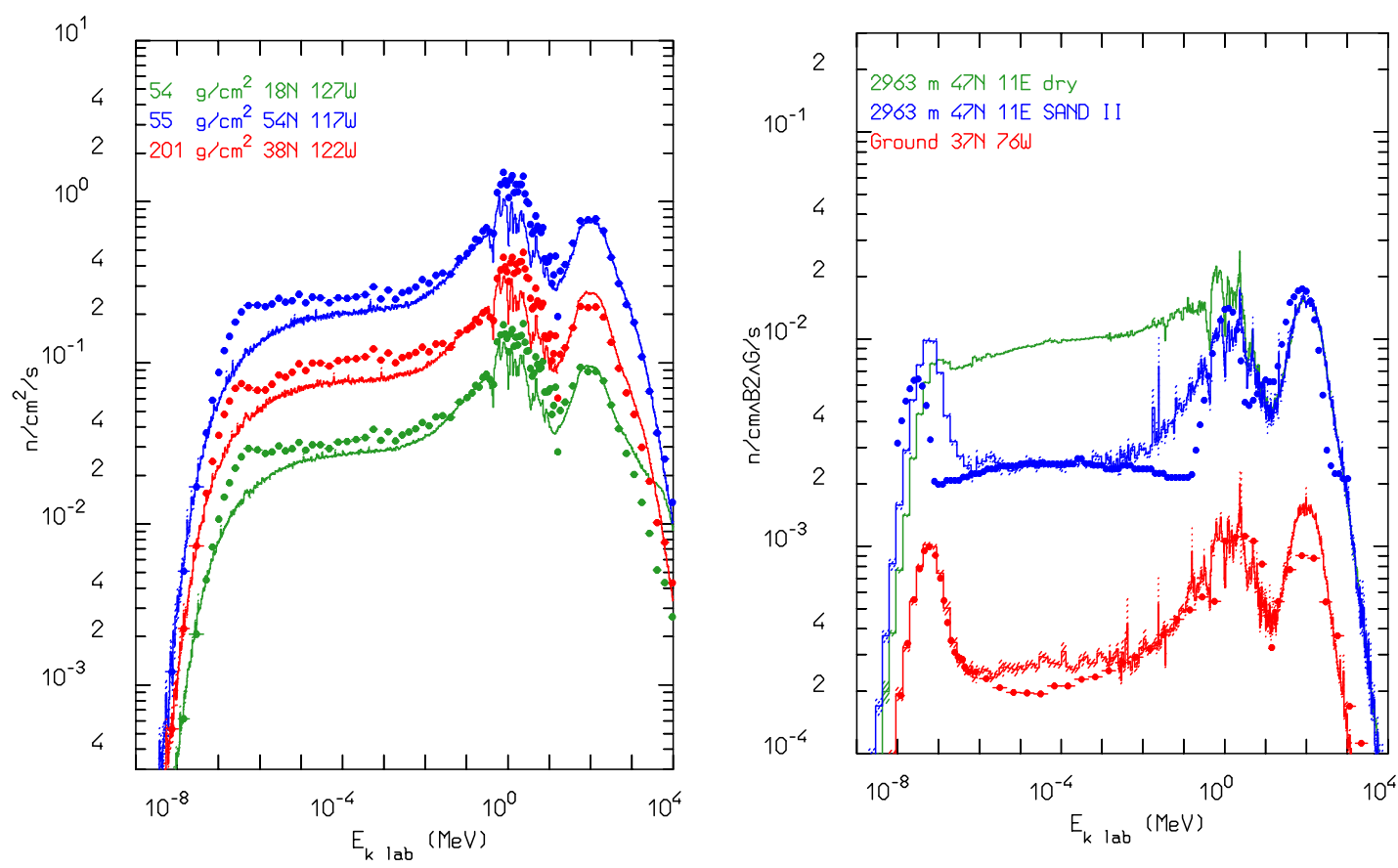

Figure 1: Left: FLUKA (line) and exp. [13] (symbols) neutron spectra at three depths and locations. Right: FLUKA (line) and exp. (symbols) neutron spectra at Zugspitze [14] (green for a wholly dry layout, blue under the assumptions described in the text), and at ground level [13] (red). The dashed areas represent the statistical errors of the calculation.

Another source of uncertainty arises from the limited knowledge of the precise conditions in which the data were taken. In particular, the air humidity or lack thereof and the ground composition and water content can greatly affect the shape and intensity of the low energy part of the neutron spectra. For the data at ground level of Ref. [13], concrete as ground material and 50\% air humidity was assumed, while for the data of Ref. [14] a mix of soil and snow and saturated air humidity was taken, knowing that during the measurements it was raining and there was snow on the ground.

Given these limitations, the measured data are well reproduced by the FLUKA simulations. 


\section{Simulation method for air showers}

The calculations have been performed for vertical incidence, at three different primary energies, $5.6 \times 10^{14} \mathrm{eV}, 5.6 \times 10^{15} \mathrm{eV}$, and $5.6 \times 10^{16} \mathrm{eV}$, for photon, proton and ${ }^{56} \mathrm{Fe}$ primaries. Hadrons and leptons have been transported down to $5 \mathrm{MeV}$, with unstable ones allowed to decay, annihilate, or being captured, with the exception of neutrons which have been transported down to $10^{-5} \mathrm{eV}$. All relevant physics processes have been activated, including electromagnetic dissociation, and photonuclear interactions. Spectra of muons and neutrons have been recorded at 5 different atmospheric depths $\left(399,492,594,878\right.$, and $\left.1033 \mathrm{~g} / \mathrm{cm}^{2}\right)$ for several radial distances and time intervals.

For the simulations shown here, the atmosphere (represented by 100 layers following the US Standard Atmosphere) has been assumed to be completely dry, and for the ground level a typical soil composition with some level of moisture has been adopted. For real cases, and/or for comparisons with experimental data, the actual air humidity and ground composition should be implemented, since they affect both the shape and the intensity of the neutron spectra at energies below a few $\mathrm{MeV}$.

\section{Results and discussion}

In the following, we show energy spectra of neutrons and compare them to those of muons. The spectra are normalized such that the area under the histograms is proportional to the number of particles.

The muon and neutron spectra produced by different primary particles of the same energy are shown in Fig. 2. The difference between the energy spectra of muons and neutrons is striking. While the muon number decreases at low energy due to muon decay, neutrons populate the full energy range down to thermal energies. The high-energy part of the neutron spectrum is fed by neutrons produced in the same hadronic interactions as pions and kaons, which give rise to muons. The feature at about $50-100 \mathrm{MeV}$ is called the quasi-elastic peak. Below $10-20 \mathrm{MeV}$, neutron propagation is best described by diffusion. The structure in the range from 0.1 to $10 \mathrm{MeV}$ is caused by many resonance cross section channels that depend on the target material. In an ideal scenario, the energy spectrum below this resonance region follows an $\mathrm{d} N / \mathrm{d} E \sim E^{-1}$ spectrum. The lower end of the neutron spectrum is given by the thermal peak that is present if the target material contains many protons (which is the case only for the ground level because of the soil at $1033 \mathrm{~g} / \mathrm{cm}^{2}$ ).

While the expected difference in the muon number for different primary particles is also reproduced in our simulations, the neutron spectra of protons and iron nuclei are very similar at high energy. In contrast, the number of neutrons in photon showers is much lower. This is a numerical coincidence that also approximately holds at the other energies considered here. It is related to the attenuation of neutrons in the atmosphere and is illustrated by the comparisons shown in Fig. 3. The muon flux does not significantly decrease at depth values larger than the shower maximum. But the number of neutrons decreases by nuclear interactions and energy losses due to quasi-elastic scattering. The so-called neutron removal length is estimated to be of the order of $100 \mathrm{~g} / \mathrm{cm}^{2}$. In fact, the originally larger production rate of neutrons in iron showers, similar to that of muons, is not visible because the iron showers have a shower maximum that is about $120 \mathrm{~g} / \mathrm{cm}^{2}$ lower than that of protons. The additional depth from the maximum to the observation level that 

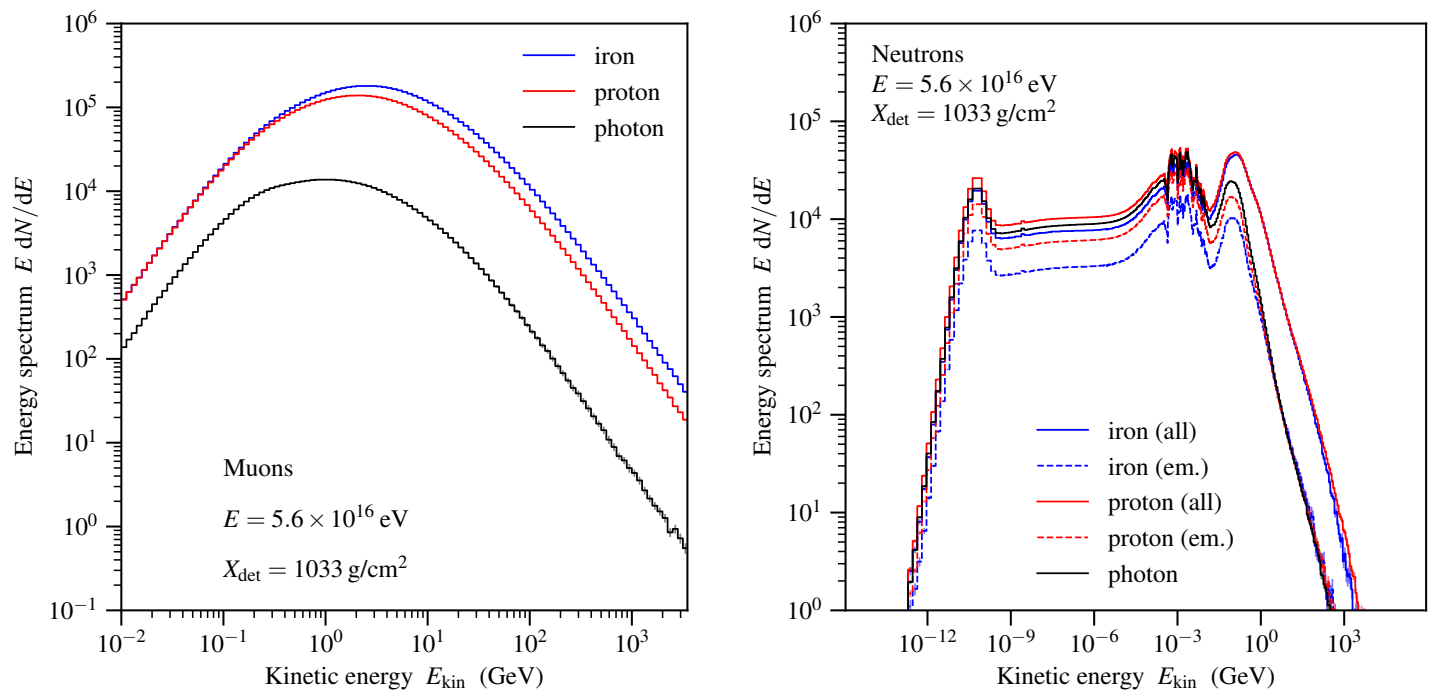

Figure 2: Muon and neutron energy spectra at sea level (directly above the soil). The results are shown for different primary particles. In addition, the neutrons produced solely by photons in air showers (mainly em. dissociation of target nuclei) are shown by dashed histograms.
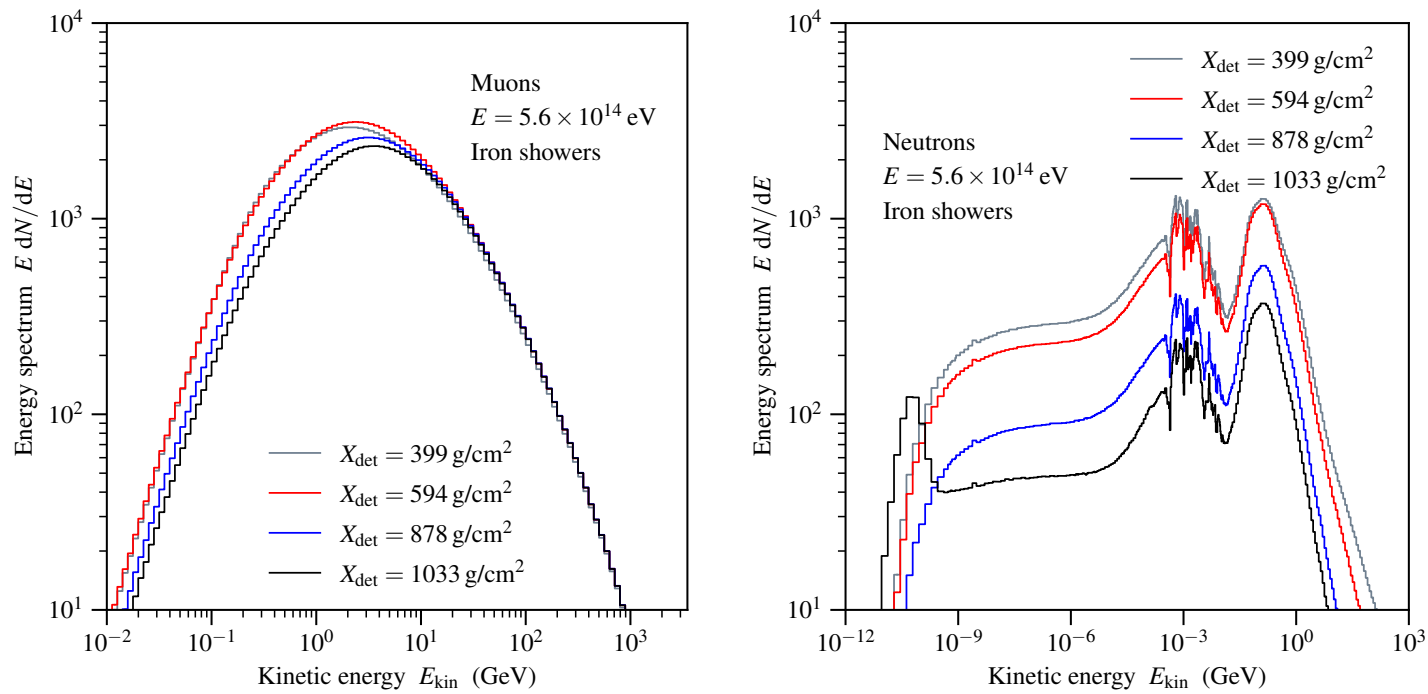

Figure 3: Muon and neutron energy spectra at different depths. The neutron energy spectrum undergoes a much stronger attenuation than the muon spectrum, which is hardly attenuated at high energy.

neutrons have to traverse in the case of iron showers makes the high-energy neutron flux of proton and iron showers numerically very similar.

The scaling of the secondary particle spectra with the primary energy is shown in Fig. 4. As expected from the Matthews-Heitler model [15], the number of muons increases only $\sim E^{\beta}$ with $\beta \sim 0.9$. After linearly re-scaling with the primary energy, the muon flux of the highest 

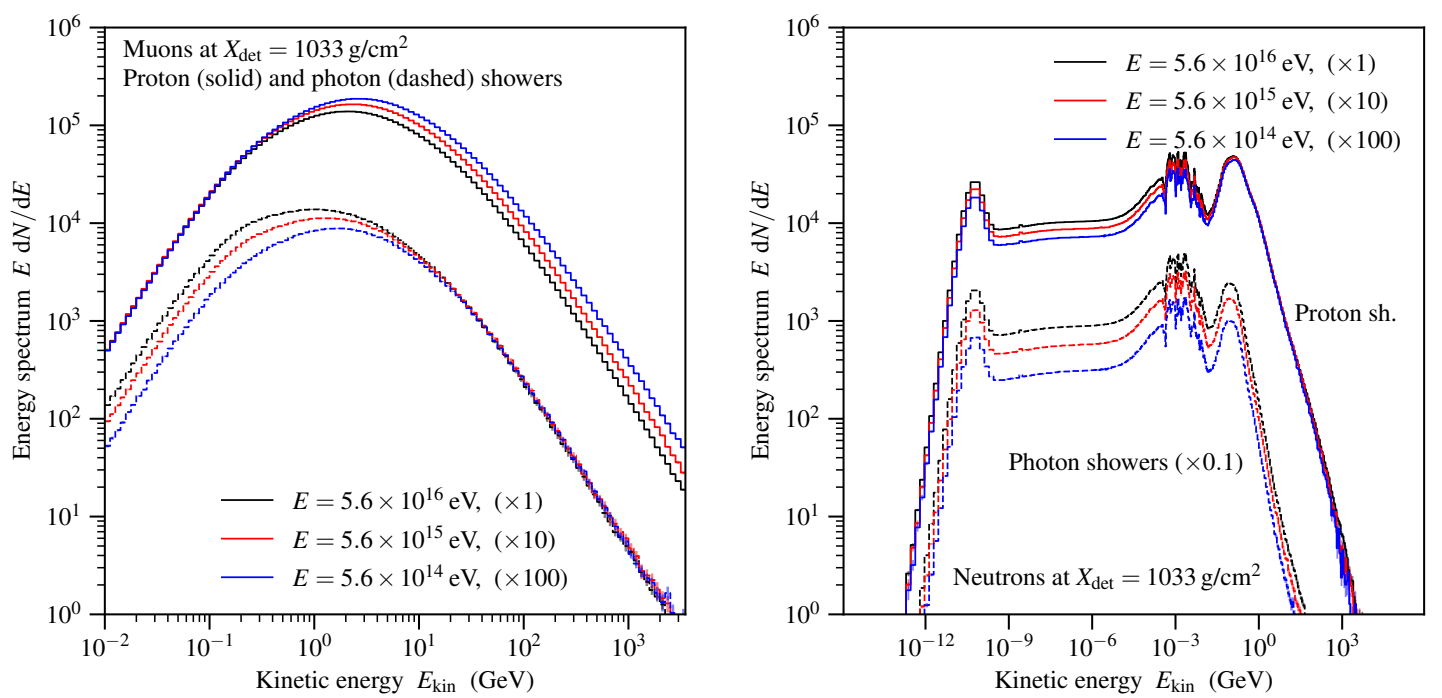

Figure 4: Scaling of secondary particle spectra with primary energy. The spectra are shown for proton and photon showers and multiplied by the factorss that would align the spectra if they scale linearly with primary energy.

primary energy is lower than that of the lower primary energy. In contrast, muon production in photon showers scales linearly with energy, again in agreement from the expectations of the Heitler splitting model. Surprisingly, such an energy scaling seems also to be present in the case of highenergy neutrons. This is, however, again the result of two effects. Firstly, the number of neutrons increases with energy in the same way as for muons. Secondly, the shift of higher-energy showers deeper into the atmosphere, which does not matter for muons, reduces the attenuation on neutrons and, hence, additionally increases the number of neutrons at ground. Effectively, a scaling of the neutron flux (at high energy only) approximately proportional to the shower energy is found for observation depths deeper than the shower maximum.

The time delay distributions of muons and neutrons relative to particles propagating with the speed of light from the first interaction point of a shower to the observation point are shown in Fig. 5. While the bulk of muons arrive with a time delay of not more than $100 \mathrm{~ns}$, this time is increased for neutrons to $10^{4} \mathrm{~ns}\left(E_{\mathrm{kin}}>20 \mathrm{MeV}\right)$ and delay times of $10^{8} \mathrm{~ns}$ are typical for neutrons of the lowest energies.

To illustrate the potential importance of neutron signals in air shower measurements, we show the time delay distribution for muons and neutrons together for an observation distance of more than $400 \mathrm{~m}$ in Fig. 6. At a depth of $594 \mathrm{~g} / \mathrm{cm}^{2}$, not far from the shower maximum, the number of neutrons becomes even comparable to that of muons. This is related to the high efficiency of neutrons to be scattered to large angles, leading to a very wide lateral distribution. The bulk of neutrons with $E_{\text {kin }}>20 \mathrm{MeV}$ arrive, in general, with time delays of $1-20 \mu$ s relative to the shower front. These properties qualitatively agree with those reported by Linsley [2] and others.

Production and, in particular, propagation of neutrons with energies of the order of $10-100 \mathrm{MeV}$ or lower are typically not simulated in modern air shower programs. Whether these neutrons lead 

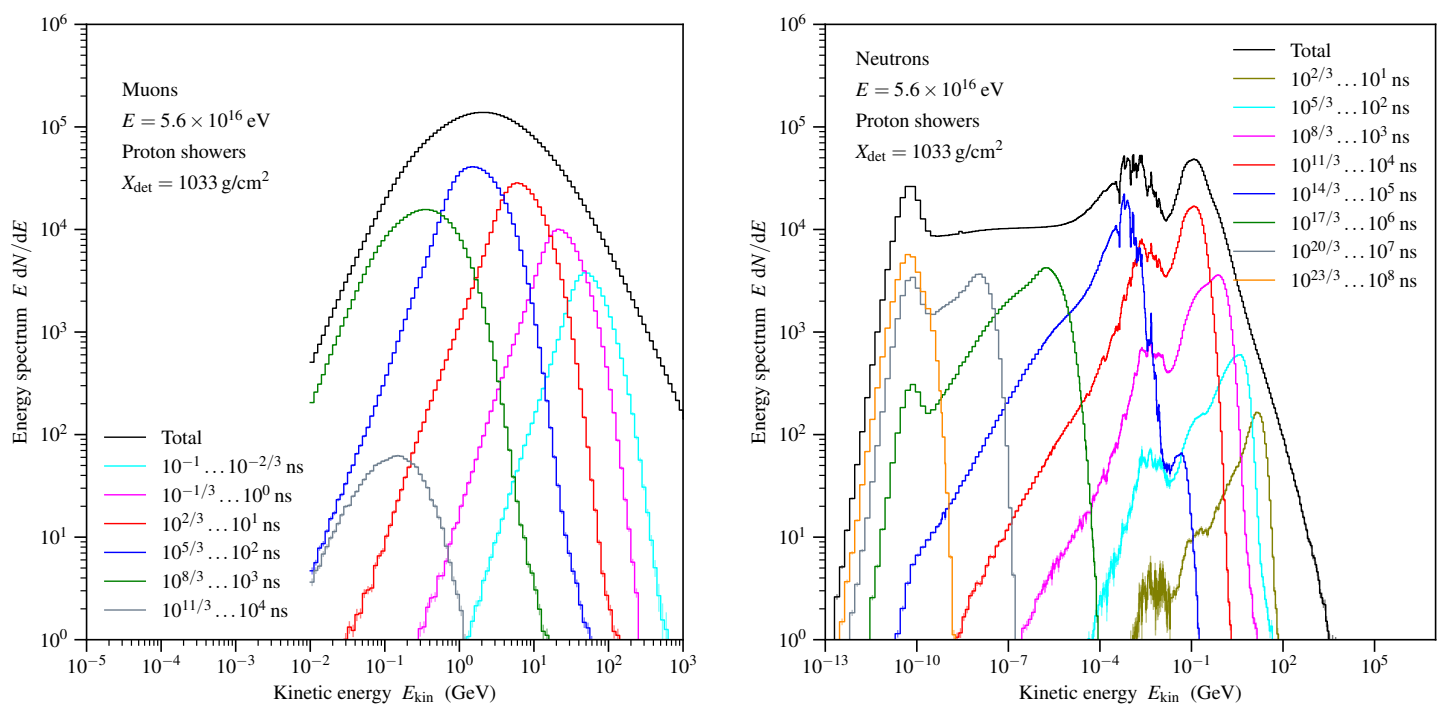

Figure 5: Energy spectra and time delay distributions of muons and neutrons at a depth of $1033 \mathrm{~g} / \mathrm{cm}^{2}$. For clarity, only a subset of the logarithmically evenly spaced time intervals are shown.
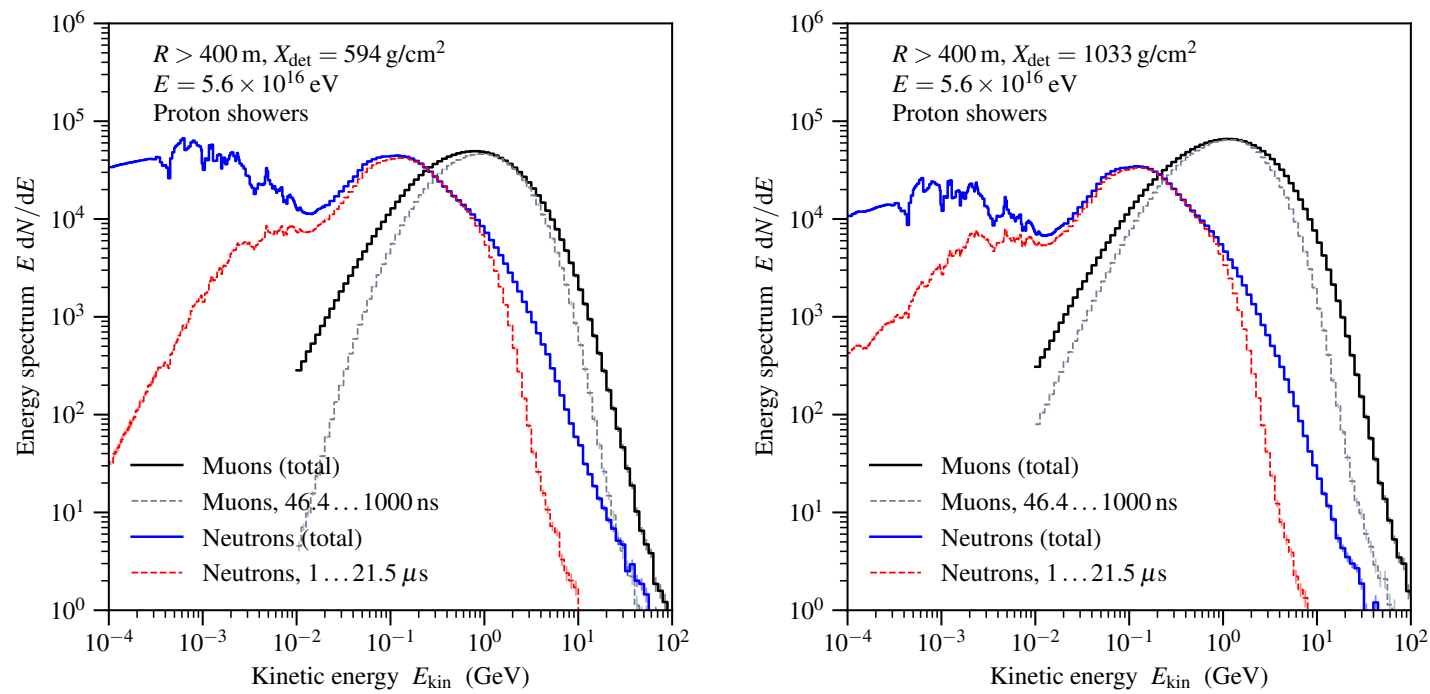

Figure 6: Energy spectra and time delays of muons and neutrons at large lateral distance and different observation depths. Relative to the number of muons, neutrons are most abundant at large lateral distance and at depths not much larger than that of the shower maximum.

to abundant or rare late pulses, or possibly even modify the integrated shower signal significantly depends on the employed detector types, detection geometry, and ambient media. The investigation of these questions is beyond the scope of this contribution and will be addressed in the future. 
Acknowledgements It is our pleasure to acknowledge very fruitful and motivating discussions with our colleagues of the Pierre Auger Collaboration. We thank Alan Watson who has drawn our attention to the early work of Linsley and Hillas on this subject and provided us with an unpublished note by Hillas on neutron production and its relevance for different air shower detectors.

\section{References}

[1] V. Tongiorgi, Neutrons in the Extensive Air Showers of the Cosmic Radiation, Phys. Rev. 75 (1949) 1532-1540.

[2] J. Linsley, Subluminal pulses from cosmic ray air showers, J. Phys. G 10 (1984) L191-L195.

[3] A. Shepetov et al., Measurements of the low-energy neutron and gamma ray accompaniment of extensive air showers in the knee region of primary cosmic ray spectrum, Eur. Phys. J. Plus 135 (2020) 96.

[4] Y. V. Stenkin, D. D. Dzhappuev, and J. F. Valdes-Galicia, Neutrons in extensive air showers, Phys. Atom. Nucl. 70 (2007) 1088-1099.

[5] A. D. Erlykin, The neutron 'thunder' accompanying large extensive air showers, Nucl. Phys. B Proc. Suppl. 175-176 (2008) 330-333.

[6] A. Ferrari, P. R. Sala, A. Fasso, and J. Ranft, FLUKA: A multi-particle transport code (Program version 2005), 2005 CERN-2005-010.

[7] T. T. Bohlen, F. Cerutti, M. P. W. Chin, A. Fassò, A. Ferrari, P. G. Ortega, A. Mairani, P. R. Sala, G. Smirnov, and V. Vlachoudis, The FLUKA Code: Developments and Challenges for High Energy and Medical Applications, Nuclear Data Sheets 120 (2014) 211.

[8] A. Ferrari and P. R. Sala, The Physics of High Energy Reactions, in proceedings of the Workshop on Nuclear Reaction Data and Nuclear Reactors Physics, Design and Safety, Miramare-Trieste, Italy, 15 April-17 May 1996, World Scientific, A. Gandini, G. Reffo eds. vol. 2 (1998) 424.

[9] A. Fedynitch and R. Engel, Revision of the high energy hadronic interaction models PHOJET/DPMJETIII, Proc. $14^{\text {th }}$ Int. Conf. on Nuclear Reaction Mechanisms, Varenna, Italy, 15-19 June 2015, CERNProceedings 2015-001 (2015) 291.

[10] S. Roesler, R. Engel, and J. Ranft, The Monte Carlo event generator DPMJET-III, in Proc. of Int. Conf. on Advanced Monte Carlo for Radiation Physics, Particle Transport Simulation and Applications (MC 2000), Lisbon, Portugal, 23-26 Oct 2000, A. Kling, F. Barao, M. Nakagawa, L. Tavora, P. Vaz eds., Springer-Verlag Berlin, p. 1033-1038 (2001), 2000.

[11] H. Sorge, Flavor production in $\mathrm{Pb}(160 \mathrm{~A} \mathrm{GeV})$ on Pb collisions: Effect of color ropes and hadronic rescattering, Phys. Rev. C52 (1995) 3291.

[12] F. Cerutti, G. Battistoni, G. Capezzali, P. Colleoni, A. Ferrari, E. Gadioli, A. Mairani, and A. Pepe, Low energy Nucleus-Nucleus reactions: the BME approach and its interface with FLUKA, Proc. $11^{\text {th }}$ International Conference on Nuclear Reaction Mechanisms, Varenna, Italy, 12-16 June 2006, E. Gadioli ed. (2006) 507.

[13] P. Goldhagen, M. Reginatto, T. Kniss, J. W. Wilson, R. C. Singleterry, I. W. Jones, and W. Van Steveninck, Measurement of the energy spectrum of cosmic-ray induced neutrons aboard an ER-2 high-altitude airplane, Nucl. Inst. Meth. A476 (2002) 42.

[14] H. Schraube, J. Jakes, A. Sannikov, E. Weitzenegger, S. Roesler, and W. Heinrich, The Cosmic Ray Induced Neutron Spectrum at the Summit of the Zugspitze (2963m), Radiat. Prot. Dosim. 70(4) (1997) 405.

[15] J. Matthews, A Heitler model of extensive air showers, Astropart. Phys. 22 (2005) 387-397. 